\title{
Resource Allocation Models for Allocating Scarce Resources: A Rapid Review to Inform Referral in Pediatric Healthcare System
}

\author{
Maurine Awuor Onyango \\ Jaramogi Oginga Odinga \\ University of Science and \\ Technology, Kenya
}

\author{
Joshua Agola \\ Jaramogi Oginga Odinga \\ University of Science and \\ Technology, Kenya
}

\author{
Samuel Liyala \\ Jaramogi Oginga Odinga \\ University of Science and \\ Technology, Kenya
}

\begin{abstract}
Referral system plays a vital role in management of diseases in any healthcare system. However, unfavorable distribution of healthcare resources continues to hamper delivery of services particularly to pediatric patients, with estimated global mortality of 5 million every year. Whereas models for resource allocation for situating scarce resources are sufficiently documented, models for allocating resources for improving healthcare systems for pediatric patients are limited.

The authors conducted a rapid review of different models for allocating scarce resources during various situations such as emergencies, epidemics and pandemics. The researchers searched Medline, EMBASE, BMC, Open Access Journals and SCOPUS from April 2005 to April 2021. They also conducted full-text screening and data abstraction independently and in duplicate. Articles were included if they were primary research, focused on models for resource allocation for a particular situation, and the model described was related to areferral system. The paper summarizes each model including their elements and operating characteristics.
\end{abstract}

From 1,542 unique citations, 295 full-text articles were reviewed and 12 articles were included for analysis. Seven studies described critical emergency situations or pandemics and five described ethical models for various ailments. Of these, four studies described both protocols and ethical model for resource allocation during Covid 19. Six articles described unique triage or model criteria (two algorithm-based, three point-based) for resource allocation. Three described referral criteria, while three point-based models were good predictors of referral for improving healthcare system for pediatric patients.

This review summarizes available resource allocation models to provide decision-makers with data to help select and tailor allocation of healthcare resources, considered scarce. Given the uncertainty shrouding pediatric diseases and resource availability in healthcare systems especially in the developing countries, jurisdictions should prepare by selecting and adapting a model that works best for their circumstances.

\section{Keywords}

Resource allocation models; Healthcare resources; Covid 19; pediatric patients; Healthcare systems; Patient referral

\section{INTRODUCTION}

To allocate scarce resources among distributed healthcare users is a challenge for all health systems management globally (1 - 4). Decisions regarding allocation of healthcare resources without a model based on some responsibly structured model may result in marked patient-to-patient variability (5 - 6). Healthcare systems, denoted by sparsely located facilities, are loosely connected with undefined interdependencies in their software models, hence limiting the scope of optimization of distributed resources (7). In turn, nurse scheduling, patient appointment, medical resource planning and reallocation, emergency medical services, disease screening policy and hospital collaboration have remained poor (8). Resource allocation models so far documented have aimed at solving problems associated with allocating scarce resources during pandemics and emergencies, overlooking referral processes particularly for pediatric patients.

Resource allocation refers to the allocation of resources to a service, department or project (9 - 10). Resource allocation models in healthcare discussion have been adequately documented. Knebel et al (11) offered an ethical model and logic model for decision making during catastrophic disasters. The authors adapted medical triage and the federalism principle to the decision-making process for allocating scarce federal public health and medical resources. Timbie et al (12) developed and validated an algorithm to guide selection of patients for pediatric critical care admission during a severe pandemic when Crisis Standards of Care are implemented. Scott et al (9) explored allocative efficiency tools for guiding budget allocation decision in malaria funding in Nigeria. Lin, Taylor, and Cohen (13) developed a disaster triage tool for the evacuation of hospitalized neonatal and pediatric populations. They assessed inpatients using bedside visual assessments and chart review to categorize patients transport level based on local emergency medical services protocols and expert opinion. Naidoo and Naidoo (14) proposed a model for regional triage committees adapted to the South African context. The model consisted of the formation of Provincial multi-disciplinary Critical Care Triage Committees to alleviate the emotional, moral and legal burden on individual ICU teams and co-ordinate inter-facility collaboration using an adapted model. The foregoing studies suggest that resource allocation models for allocating scarce resources especially for improving pediatric patients' healthcare are scanty.

According to Yore et al (15), pediatric patients account for $20-35 \%$ of all emergency hospital visits globally. Moreover, children present a unique distribution of chief health complications compared to adults and require diverse clinical management (16). Housseine et al (17) posit that with over 5 million deaths every year, perinatal death remains a significant global health problem. The World Health 
Organization (18) on their part reports that countries with the highest absolute numbers of stillbirths and neonatal deaths are in Asia and sub-Saharan Africa. Despite of tremendous reduction in mortality rate of children under 5 years, the Europe PMC Funders Group (19) report that 5.8 million children in this cohort died in 2015 globally. Neonatal deaths is estimated to account for $53.1 \%$ of the total under-5 deaths each year (20). Provision of healthcare to pediatric patients therefore seems to be a major challenge particularly in the Sub Saharan Africa including Kenya. This in turn demands for the understanding of effectiveness of models associated with resource allocation for pediatric patient referral improvement. One of the approaches adopted for improving access to quality healthcare is referral (21). A referral is a process in which a health worker at a one level of the health system, having insufficient resources (drugs, equipment, skills) to manage a clinical condition, seeks the help of a better or differently resourced facility at the same or higher level (22). According to Francetic, Tediosi and Kuwawenaruwa (23), the role of referrals in obstetric and childcare is crucial for the effectiveness of antenatal care but also for pediatric patients. Researchers $(23$ - 29) have reported that a high risk of child and maternal mortality is directly associated with inefficiencies in the referral system. However, challenges such as infrastructure, health information systems, capacity of health care workers, and financial resources often bedevil implementation of health care referral system in the developing countries such as Kenya $(26-29)$. Therefore, efforts aimed at strengthening referral system endeavor to manage pediatric patients' health needs comprehensively by using resources beyond those available where they access care are imperative. To this end, in this review, we sought to identify, classify and assess the types of resource allocation models focused on improving referral for pediatric patients' health system.

\section{METHODS}

The study conducted a rapid literature review covering resource allocation models for allocating health resources, and referral systems for pediatric patients. This was done in line with the widely accepted Preferred Reporting Items for Systematic Review and Meta-analysis statement (PRISMA), as prescribed by Moher et al (30 - 32). Further, the guidelines proposed by Khan et al (34) i.e. (1) Framing the question; (2) Identifying relevant publications; (3) Assessing study quality; (4) Summarising the evidence; and (5) Interpreting the findings were adopted. The researchers begun by doing a simple search in Google Scholar using terms such as resource allocation models, resource allocation models for allocating healthcare resources, models for allocating resources in referral healthcare systems, and resource allocation models in referral systems for pediatric patients. Further, the researchers searched Medline, EMBASE, BMC, Open Access Journals and SCOPUS. Eligibility criteria was based on studies geared towards answering the question as to how resource allocation models aid in allocating scarce resources in healthcare referral system for pediatric patients (31). In addition, articles were included if they were primary research, focused on models for resource allocation for a particular situation, and the model described was related to a distributed or referral system. To ensure data quality, the researchers conducted full-text screening and data abstraction independently and in duplicate (32). The acronym PICOT informed the eligibility criteria for inclusion in the systematic review: the population (participants) of focus, types of interventions (and comparisons), and the outcomes of interest. The time relates to the period within which the studies were published (Table
1). The paper summarizes each model including their elements and operating characteristics.

Table 1: PICOT Based inclusion criteria

Patient Population Pediatric patients (children under 5 years)

Interventionof interest Allocation of resources guided by resource allocation model

Comparison None

interventions

Outcomes:

Primary Successful referral of pediatric

Outcome patients

Secondary Quality healthcare services and treatment, and good health

Outcome

Time

$2005-2021$

Otherconsiderations English (Language)

The quality of the included articles was rated as either "poor", "fair" or "good" by three independent researchers (EO, MA' and $\mathrm{ZO}$ ), and EO made the final adjudication in cases of nonagreement. The rating of the articles was based on the criteria provided by Moher et al [30].

\subsection{Data extraction}

The data were extracted using an excel spreadsheet under the following headings: study setting, sample characteristics, intervention objectives, study design and methods, outcome measures and results.

\subsection{Data analysis}

We employed a thematic content analysis approach to distil information from the selected articles (34). The extracted information was coded into two broad categories: Intervention components and outcomes measured as informed by the study aim. The intervention components were classified along as: (1) How the interventions were administered; (2) who delivered the intervention; (3) Point of intervention delivery; and (4) components of the intervention. The outcomes were coded according to the reported primary and secondary outcomes of the study.

\section{RESULTS}

The literature search identified 2,591 unique citations (Fig. 1). After assessing titles and abstracts for eligibility, 1, 542 articles were included for full-text review. After screening for potentially relevant titles and abstracts 1,247 articles were excluded. After screening full-texts, 283 papers were further excluded because they did not report on resource allocation models for allocating scarce healthcare resources during emergencies of for enhancing referral for pediatric patients. Subsequently, 12 papers were included in the review for final analysis. 


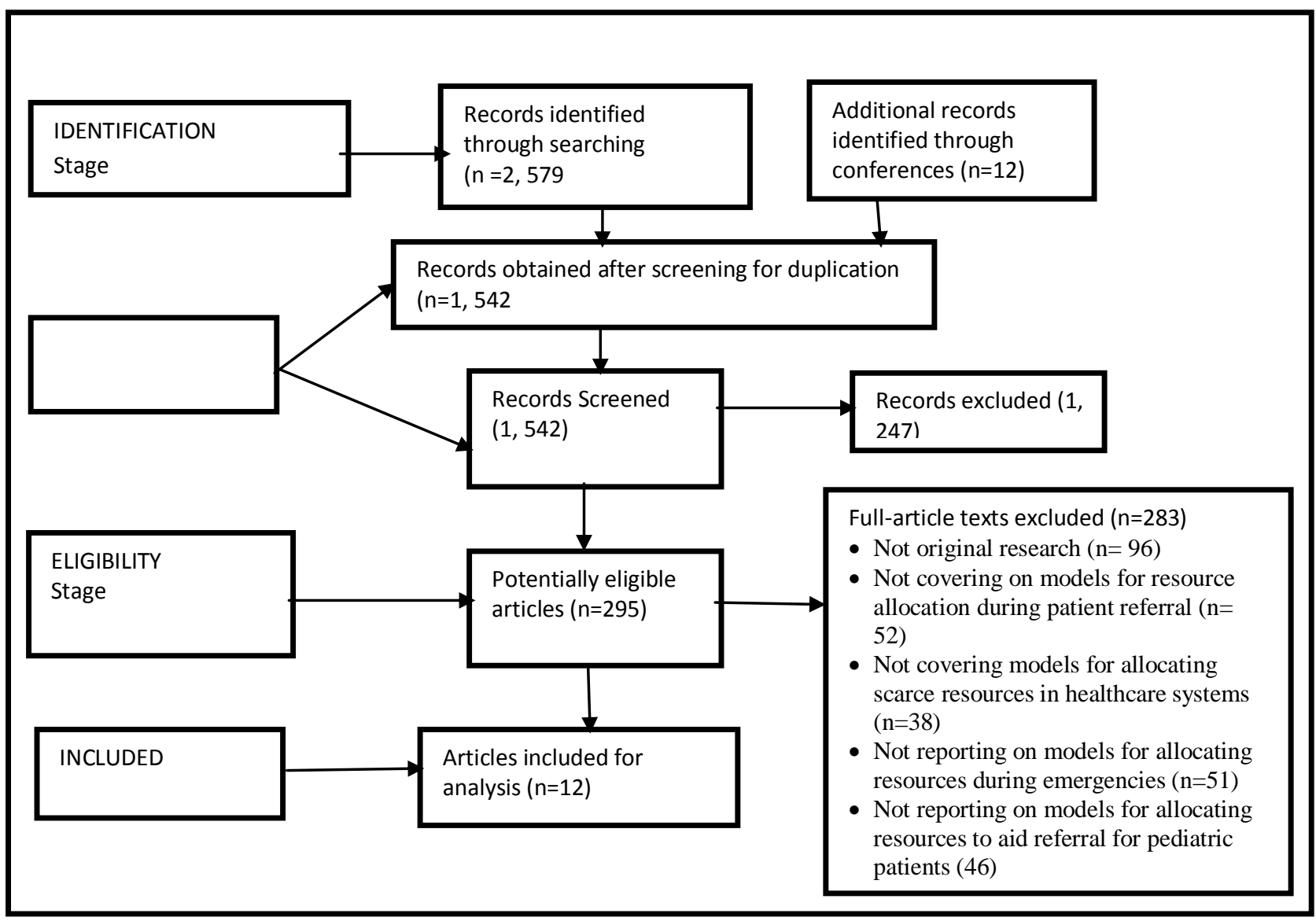

Figure 1: The Preferred Reporting Items for Systematic Reviews and Meta-Analyses (PRISMA) flow protocol of study selection

\subsection{Characteristics of included studies}

The characteristics of the studies included in the review are summarized in Table 2. The 12 papers studies were conducted between 2012 and 2021. Four of the studies were conducted in United States of America (11. 12, 35, 36), two were conducted in South Africa $(14,37)$, while one study was done each in Italy, Taiwan, UK, Kenya, and Malawi (1, 8, 13, 29, 38). Six of the papers were retrospective cohort studies (12, $15,35,37,39,40)$, four randomized control trials (RCT) $(1$, $11,13,14)$, two mixed method involving qualitative and quantitative approaches $(29,38)$ and one system simulation study (8).

Table 2: Characteristics of included studies (n=12)

\begin{tabular}{lcl}
\hline Characteristics & Counts & References \\
\hline $\begin{array}{l}\text { Year of } \\
\text { publication }\end{array}$ & & \\
20052011 & 0 & \\
$2011-2021$ & 12 & $\begin{array}{l}1,2,8,11,12,13,14,15, \\
29,35,36,37,38\end{array}$
\end{tabular}

\section{Country}

$\begin{array}{lll}\text { USA } & 5 & 2,11.12,35,36 \\ \text { Iran } & 1 & 8 \\ \text { Cambodia } & 1 & 15\end{array}$

$\begin{array}{lll}\text { Italy } & 1 & 1 \\ \text { South Africa } & 2 & 14,37 \\ \text { UK } & 3 & 13,39,40 \\ \text { Saudi Arabia } & 1 & 38 \\ \text { Kenya } & 1 & 29 \\ \text { Study Design } & & \\ \begin{array}{l}\text { Retrospective } \\ \text { cohort }\end{array} & 5 & 12,15,35,37,39,40 \\ \begin{array}{l}\text { Randomised trial } \\ \text { Mixed method }\end{array} & 4 & 1,11,13,14 \\ \begin{array}{l}\text { (qualitative } \\ \text { Quantitative) }\end{array} & 29,38 \\ \begin{array}{l}\text { System } \\ \text { Simulation }\end{array} & 1 & 8\end{array}$

\subsection{Period of Study and Nature of Intervention}

The period of the studies was particularly during pandemic or referrals (Table 3). Seven studies evaluated resource allocation models. These include resource allocation criteria 
in a hospital, ethical model for resource allocation, strategies to allocate resources during mass casualty, triage by resource allocation for hospitalized pediatric patients, model for prioritising scarce intensive care unit resources in the midst of COVID- 19, and resource allocation for congenital cardiac catheterization during the COVID-19 pandemic $(1,11,12,13$, 14, 35, 36). Three studies evaluated provision of patient referral including simulation optimization methods for solving patient referral problems in hospital environment, challenges facing implementation of referral system for quality health care services, and provision of referral services at primary care $(8,29,38)$.

Similarly, one study evaluated the Characteristics and outcomes of pediatric patients presenting at referral hospitals (15). On the other hand, another study proposed and validated a model for pediatric self-management (36). Table 4 illustrates the period and nature of the interventions identified.

Table 3: Intervention, Study Location, and Duration of the Study

\begin{tabular}{ll}
\hline Article & Coun Period and Nature of Intervention \\
try/st \\
udy \\
locati \\
on
\end{tabular}

Bodina, et al (2017) Italy

Hassan (2019) Iran

Knebel et al (2014) USA

Timbie et al (2013) USA

Lin, Taylor and UK

Cohen (2018)

Naidoo and Naidoo South Africa (2021)

Yore et al (2018) Cambodia

Nango et al (2017) Kenya Resource allocation criteria in a hospital

Artificial bee colony allocation model disasters
Framework for prioritising scarce intensive care unit resources in the midst of COVID- 19

Characteristics outcomes of pediatric patients presenting referral hospitals

Determinants of a functional referral system in Kisumu County, Kenya patients' perspective
Morray et al (2021) USA

Modi et al (2012)

USA

Nkonki et al (2017) South Africa

Alkinaidri and

Alsulami (2018)
Resource allocation for congenital cardiac catheterization during the COVID-19 pandemic

A Framework for pediatric selfmanagement

Modelling the cost of community interventions to reduce child mortality

Improving Healthcare Referral System Using Lean Six Sigma

Resource allocation strategies or models during emergencies and other relevant topics formed an integral part of all 12 interventions $(1,2,8,11,12,13,14,15,29,35,36,37$, and 38 ). The resource allocation models discussed in the analysed studies were developed to solve the problem of distributing scarce resources during severe epidemics like Covid 19 (14, $35)$. Other resource allocation models were also developed to aid distribution of healthcare resources during various emergencies or disasters $(1,11,12$, and 14). Similarly, the interventions also presented studies related to optimization strategies or methods for solving the problems associated with algorithm for solving patient referral $(8,15,29$, and 38). Finally, some of the fuzzy multi-objective bed analysed studies focused on resource allocation models for pediatric patients particularly during emergencies and referrals $(13,35,36$, and 37$)$.

An ethical framework for Similarly, two studies $(12,13)$ specifically used in-patient allocating scarce medical pediatric data (retrospective design) to formulate two models resources to states during (simulation and disaster triage tool respectively) for determining diverse health indicators of children. In one study (12), Discrete Event Simulation was used to Strategies to manage and determine triage thresholds for probability of death and allocate scarce resources duration of ventilation as a function of casualty volume and during mass casualty the total number of available beds. Simulation was employed events to compare survival between the proposed triage algorithm and a first come first served distribution of scarce resources.

Triage by Resource Greater survival of population was realised using Allocation for the triage thresholds compared with a first come first served hospitalized pediatric strategy. In this model, for five, six, seven, eight, and 10 patients thousand casualties, the triage algorithm increased the number of lives saved by $284,386,547,746$, and 1,089 , respectively, compared with first come first served. In the other study, a disaster triage tool for the evacuation of hospitalized neonatal and pediatric populations was developed (13). This was employed to assess pediatric inpatients using bedside visual assessments and chart review to categorize patients transport level based on local emergency medical services protocols

and and expert opinion. Primary outcome was the number of each level of transport required for hospital evacuation. Secondary at outcome was improved efficiency of obtaining information about specific transport needs for evacuation.

Two studies $(14,35)$ proposed resource allocation models for distributing scarce healthcare resources during the recent pandemic that continues to ravage the world to date, the Corona Virus (14). A model for regional triage committees adapted to the South African context consisting of the formation of Provincial multi-disciplinary Critical Care Triage 
Committees was formed to alleviate the emotional, moral and legal burden on individual ICU teams and co-ordinate interfacility collaboration using an adapted model (14). The mandate of the committee comprised of an impartial, broader and ethically sound viewpoint with time to consider broader contextual factors such as adjusting rationing criteria according to different levels of pandemic demand and the latest clinical evidence. Direct feedback to national level and accountability to a national monitoring committee strengthened the services of the committee. The study observed that no single ethical approach to decision-making is sufficient regarding resource allocation during Covid 19; instead, one, which considers multiple contextual factors, is necessary. Another study defined current patterns and provided guidance and recommendations on the preservation and repurposing of resources to help pediatric cardiac programs develop strategies for patient care during COVID19 pandemic (35). The study found a relative uniformity among centers as to which procedures were considered elective. Only three centers had performed a catheterization on a confirmed COVID-19 positive patient. Centers located in areas with a higher number of COVID-9 cases have been more involved in a simulation of donning and doffing personal protective equipment (PPE) than low-prevalence centers.

Patient referral including models formulated for enhancing pediatric referral and associated challenges are discussed among the analysed articles (8, 15, 29, and 38). System simulation was used to construct a model and develop a simulation optimization method, combining the heuristic algorithm (patient referral mechanism) with the particle swarm optimization (PSO) method, to determine a better way to refer patients from one hospital (referring hospital) to another (recipient hospital) to receive certain imaging services (8). After the simulated model was verified and validated, three patient referral mechanisms to dispatch referring patients to the appropriate recipient hospitals were proposed. Based on the numerical results, the findings showed that Mechanism 2, transferring patients to the hospital with the shortest waiting time, had good performance in both scenarios: allowing patient referrals among all hospitals and limiting the patients' waiting time.

Another study observed the characteristics and outcomes of pediatric patients presenting at Cambodian referral hospitals without appointments (15). Predictors of admission included transfer or referral from another health provider, seeking prior care for the presenting problem, low socioeconomic status, onset of symptoms within $24 \mathrm{~h}$ of seeking care, abnormal vital signs or temperature, and chief complaint of abdominal pain or fever. Similarly, challenges facing implementation of the referral system for quality health care services in Kenya was analysed (29). The study revealed that infrastructure, health information systems, capacity of health care workers, and financial resources were challenges in implementation of health care referral system. Another study investigated the factors that affect management of the referral system at the primary level of health care in Saudi Arabia (38). It showed that primary care facilities faced a number of challenges such as lack of referral transport, poor communication, lack of referral guidelines, shortage of skilled personnel, lack of diagnostic equipment and drugs, leading to the self-referral and the bypassing of primary care facilities.

The systematic review also revealed that only one study looked at pediatric mortalities and associated models to solve the problem (37). The study used the Lives Saved Tool (LiST), a model in the spectrum software, to estimate the costs and impact on reducing child mortality of scaling up interventions delivered by community health workers at community level from a provider's perspective. Results showed that a total of 9 interventions can prevent 8891 deaths by 2030 . Hand washing with soap $(21 \%)$ accounts for the highest number of deaths prevented, followed by therapeutic feeding (19\%) and oral rehydration therapy (16\%). The top 5 interventions account for $77 \%$ of all deaths prevented.

\subsection{Discussions}

This systematic review makes a unique contribution due to its explicit focus on models used for resource allocation decision making for scarce healthcare resources, with an aim of solving the problem of pediatric patient referral. The literature synthesised suggests that along with models based on algorithms, ethical considerations relating to cost saving, socio economic considerations, equity, patients and health systems play a role in the resource allocation decisionmaking. While it may not be fully possible to specifically highlight how resource allocation models have influenced referral of pediatric patients, it is worth isolating and examining them to understand how they have the potential to influence referral decision-making.

Variation of referral patterns and rates has drawn attention for some time now, although reasons for this are not fully understood, indicating they are varied, idiosyncratic and integral to the context. One of the notable findings is the small number of included studies, despite the inclusion of a broad date range, suggesting the impact of poor resource allocation decision making when referring pediatric patients for special care.

As highlighted by this review, a myriad of studies focusing on models for resource allocation have been conducted in response to crisis occasioned by pandemics. While such models may be useful in aiding referral processes, a huge amount of attention has been directed at Covid 19 (2, 3, 4, 6, 14). Of the studies on resource allocation models or models, distribution of mechanical ventilators is a major central focus in the discussions. This can stem from an obligation of responsibility for patients' wellbeing and unwillingness to take risks.

This systematic review has highlighted several models for allocating scarce healthcare resources. Some of the models include ethical model and logic model for decision making during catastrophic disasters. This model adapted medical triage and the federalism principle to the decision-making process for allocating scarce federal public health and medical resources (11). Another model is an algorithm to guide selection of patients for pediatric critical care admission during a severe pandemic when Crisis Standards of Care are implemented (12). Budget allocation decision in funding specific diseases has been guided based on allocative efficiency tools (9). Another model for evacuating neonatal and pediatric populations based on inpatients bedside visual assessments and chart review for categorizing patients transport level and local emergency medical services protocols as well as expert opinion (13). Besides models developed to aid resource allocation during health crisis, most models especially in Africa are triage based for minimizing disease burden (14)

This review points at the scarcity of documentations covering models for pediatric patient referral. This is in disregard of the fact that quiet a number of research have been documented on resource allocation models during specific crisis. The review has highlighted that pediatric patients account for $20-35 \%$ of all emergency hospital visits globally (15). It has also been highlighted in the review that children present a unique distribution of chief health complications compared to adults 
and require diverse clinical management (16). Similarly, this review has revealed that child mortality remains a significant global health problem, with over 5 million deaths every year (17). Asia and sub-Saharan Africa have been identified in the review as regions with the highest number of child mortality rates (18). One area that requires development of suitable models for guiding resource allocation especially scarce healthcare resources is therefore the treatment of pediatric patients $(19,20)$.

Models for enhancing patient referral processes have also been highlighted in this review $(8,15,29$, and 38). These models have been aligned to heuristic algorithm (patient referral mechanism) with the particle swarm optimization (PSO) method, to determine a better way to refer patients from one hospital (referring hospital) to another (recipient hospital) to receive certain imaging services (8). Transferring patients to the hospital with the shortest waiting time is revealed as being appropriated among the reviewed studies, for allowing patient referrals among all hospitals and limiting the patients' waiting time $(15,29)$. The success of referral processes have been enumerated in the review to include low socioeconomic status

Another study observed the characteristics and outcomes of pediatric patients presenting at Cambodian referral hospitals without appointments (15). Predictors of admission included transfer or referral from another health provider, seeking prior care for the presenting problem, low socioeconomic status, onset of symptoms within $24 \mathrm{~h}$ of seeking care, abnormal vital signs or temperature, and chief complaint of abdominal pain or fever. Similarly, challenges facing implementation of the referral system for quality infrastructure, health information systems, capacity of health care workers, and financial resources (29).

\subsection{Implications for policy and practice}

Internationally, health systems are struggling to meet the demand on emergency departments, with healthcare planners and managers endeavouring to reduce the strain by eliminating non-urgent resource utilization [3, 4]. Strengthening primary care capacity and capabilities, through strategies such as triage formation and scientifically modeled models such as bee algorithms or epistemic game theory, can contribute to decreasing child mortality especially in sub Saharan Africa $(37,38,39)$. This could address the pattern of referrals at certain times of the day and week, highlighted in this review. Enhanced paediatric training for healthcare providers who experience professional uncertainty when treating children may lower referrals expenses due to an aversion of risk. An alternative strategy could be the provision of remote consultations for caregivers to seek advice from paediatricians. Stronger recognition of resource allocation methods and their impact on pediatric referral is also essential during care giver training. Greater awareness of various influences on referral decision-making is vital to ensuring appropriate and excellent care for patients (38).

\subsection{Limitations}

The findings of this review are limited primarily by the small number of included studies and the variation in study sample size. Literature on resource allocation models is mostly based on emergency situations and not normal cases of referral, and often does not focus specifically on pediatric patients. The focus of this review was empirical studies and therefore policy documents have not been included, though it is accepted that this may shed further light on referral pathways.

\section{CONCLUSION}

The decision to refer a child to a special care is imbued with a complex interplay of a healthcare system that is able to allocate the requisite resources in distributed health facilities. Enhanced awareness of healthcare resource availability on referral decision-making is crucial to understanding patterns of pediatric unscheduled healthcare and to planning services that respond to children's needs, whilst allowing primary healthcare providers to make decisions in the best interest of the child. Literature examining referral variation is rather scarce besides being outdated, suggesting up to date research is required to account for system changes in recent years. This review has identified scope for further research, such as qualitative research with pediatric specialists, which can contribute to understanding the inter-play between primary and emergency services, pertinent in the context of rising paediatric presentations to the healthcare.

\section{ACKNOWLEDGMENTS}

I wish to sincerely thank my supervisors Dr. Joshua Agola and Dr. Samuel Liyala for their support, guidance and supervision.

I would also want to appreciate the support and encouragement from family and special friends during the tough times that I had to balance between the demands of a rigorous academic program.

\section{REFERENCES}

[1] Bodina, A., Pavan, A. and Castald, S. (2017). Resource allocation criteria in a hospital. Journal of previous medicine and hygiene, 58,184-189.

[2] Ranney, M. L., Griffeth, V. and Jha, A. K. (2020). Critical supply shortages: the need for ventilators and personal protective equipment during the COVID-19 pandemic. N Engl J Med 2020;382:e41.

[3] Antommaria AHM, Gibb TS, McGuire AL, Wolpe PR, Wynia MK, Applewhite MK, et al. (2020). Ventilator triage policies during the COVID-19 pandemic at U.S. associated with members of the association of bioethics program directors. Ann Intern Med., 173, 188-194.

[4] Piscitello GM, Kapania EM, Miller WD, Rojas JC, Siegler M, Parker WF. (2020). Variation in ventilator allocation guidelines by US state during the coronavirus disease 2019 pandemic: A systematic review. JAMA Netw Open, 3, e2012606.

[5] Hecht, R., Arias, D. and Krubiner, C. (2019). Responsible resource allocation, public health stewardship, and ethics. The Oxford Handbook of Public Health Ethics. Online Publication: DOI:10.1093/oxfordhb/9780190245191.013.72.

[6] Dar, M., Swamy, L., Gavin, D. and Theodore, A. (2021). Mechanical-ventilation supply and options for the COVID-19 Pandemic leveraging all available resources for a limited resource in a crisis. Ann Am Thorac Soc 18 (3), 408-416.

[7] Elias, J. (2018). Optimal resource allocation in networks: Optimization, Game Theoretical Models, and Algorithms. Unpublished thesis submitted to Universit'e Paris Descartes

[8] Hasan, A. L. (2019). Artificial bee colony algorithm for solving fuzzy multi-objective bed allocation model. 
Karbala international journal of modern science, 5 (4): DOI: 10.33640/2405-609X.1154.

[9] Scott, P.A., Harvey, C., Felzmann, H., Suhonen, R., Habermann, M, Papastravrou, E et al (2019). Resource allocation and rationing in nursing care: A discussion paper. Nursing Ethics 26(5) 1528-1539.

[10] Biddison, E.L.D., Gwon, H.S., Schoch-Spana, M., Regenberg, A.C., Juliano, C... Toner, E.S. et al (2018). Scarce resource allocation during disasters: A mixedmethod community engagement study. CHEST, 153(1):187-195.

[11] Knebel, A.R., Sharpe, V.A., Danis,Toomey, L.M.and Knickerbocker, D.K. (2014). Informing the gestalt: An ethical model for allocating scarce federal public health and medical resources to states during disasters. Disaster Med Public Health Prep., 8(1), 79-88.

[12] Timbie, J., Ringel, J., Fox, D., Pillemer, F., Waxman, D., Moore, M., et al. (2013). Systematic review of strategies to manage and allocate scarce resources during mass casualty events. Ann Emerg Med, 61(6):677- 689.e101. doi:10.1016/j.annemergmed.2013.02.005

[13] Lin, A., Taylor, K., and Cohen, R.S. (2018). Triage by Resource Allocation for in-patients: A novel disaster triage tool for hospitalized pediatric patients. Disaster Medicine and Public Health Preparedness: Inc. DOI: 10.1017/dmp.2017.139

[14] Naidoo, R. and Naidoo, K. (2021). Prioritising 'already-scarce' intensive care unit resources in the midst of COVID-19: A call for regional triage committees in South Africa. BMC Med Ethics, 22(28), 1 -9 .

[15] Yore, M.A., Strehlow, M.C., Yan, L.D., Pirrotta, E.A., Woods, J.L., Somontha, K., ..... Mahadevan, S.V. et al (2018). Characteristics and outcomes of pediatric patients presenting at Cambodian referral hospitals without appointments: An observational study. International Journal of Emergency Medicine 11(17), https://doi.org/10.1186/s12245-018-0172-0.

[16] Olubukunola, M. D, (2017). The ethical allocation of resources during a pediatric emergency mass critical care event. Curr Treat Options Peds, 3, 293-303

[17] Housseine, N., Snieder, A., Binsillim, M., Meguid, T., Browne, J.L. and Rijken, M.J. (2021). The application of WHO ICD-PM: Feasibility for the classification of timing and causes of perinatal deaths in a busy birth centre in a low-income country. PLOS ONE 16(1): $\mathrm{e} 0245196$. org/10.1371/journal.pone.0245196.

https://doi.

[18] World Health Organization. Every newborn: An Action Plan To End Preventable Deaths 2014. https://www.who.int/maternal_child_adolescent/newborn s/every-newborn/en/ (accessed October 6, 2021).

[19] Europe PMC Funders Group (2017). Global, regional, national, and selected subnational levels of stillbirths, neonatal, infant, and under-5 mortality, 1980-2015: A systematic analysis for the Global Burden of Disease Study 2015. Lancet, 388(10053), 1725-1774.

[20] Li, Z., Karlsson, O., Kim, R. and Subramanian, S.V. (2021). Distribution of under-5 deaths in the neonatal, post-neonatal, and childhood periods: A multi-country analysis in 64 low and middle-income countries. International Journal for Equity in Health, 20 (109): https://doi.org/10.1186/s12939-021-01449-8

[21] Kruk, M.E., Gage, A.D., Arsenault, C. et al. (2018). High-quality health systems in the Sustainable Development Goals era: time for a revolution. The Lancet Global Health, 6: e1196-252.

[22] Gupta, A.K., Talati, S., Bhattacharya, S. and Singh, A. (2017). Health system strengthening-focusing on referrals: An analysis from India. JOJ Nursing and Healthcare, 2 (4), 1 - 3.

[23] Francetic, I., Tediosi, F. and Kuwawenaruwa, A. (2021). A network analysis of patient referrals in two district health systems in Tanzania. Health Policy and Planning, $36,162-175$

[24] Atuoye, K.N., Dixon, J., Rishworth A et al. 2015. Can she make it? Transportation barriers to accessing maternal and child health care services in rural Ghana. BMC Health Services Research 15: 333.

[25] Bohn JA, Kassaye BM, Record D et al. 2016. Demographic and mortality analysis of hospitalized children at a referral hospital in Addis Ababa, Ethiopia. BMC Pediatrics 16: 168.

[26] Slusher TM, Kiragu AW, Day LT et al (2018). Pediatric critical care in resource- limited settings-overview and lessons learned. Frontiers in Pediatrics, 6 (49). doi: 10.3389/fped.2018.00049

[27] Hanson C, Gabrysch S, Mbaruku G et al. 2017. Access to maternal health services: geographical inequalities, United Republic of Tanzania. Bulletin of the World Health Organization 95: 810-20.

[28] Maluka S, Joseph C, Fitzgerald S, Salim R, Kamuzora P. 2020. Why do pregnant women in Iringa region in Tanzania start antenatal care late? A qualitative analysis. BMC Pregnancy and Childbirth, 20: 126.

[29] Nango, O.W., Mwangi, E.M. and Oluoch, M. (2019). Determinants of a functional referral system in Kisumu County, Kenya patients' perspective. Global Journal of Health Sciences, 4 (1), $11-31$.

[30] Moher, D., Liberati, A., Tetzlaff, J., Altman, D.G., Group, P. (2009). Preferred reporting items for systematic reviews and meta-analyses: the PRISMA statement. BMJ, 339, 2535.

[31] Tawfik, G.M., Dila, K.A.S., Mohamed, M.Y.S., Tam, D.N.H., Kien, N.D.....Huy, N.T. et al (2019). A step-bystep guide for conducting a systematic review and metaanalysis with simulation data. Tropical Medicine and Health, 47 (46): https://doi.org/10.1186/s41182-0190165-6.

[32] Snyder, H. (2019). Literature review as a research methodology: An overview and guidelines. Journal of Business Research, 104 (2019) 333-339.

[33] Fiest, K.M., Krewulak, K.D., Plotnikoff, K.M., Kemp, L.G., Parhar, K.J.S., Niven, D.J... Leigh et al (2020). Allocation of intensive care resources during an infectious disease outbreak: A rapid review to inform practice. BMC Medicine, 18 (404), https://doi.org/10.1186/s12916-020-01871-9 
[34] Snilstveit B, Oliver S, Vojtkova M. Narrative approaches to systematic review and synthesis of evidence for international development policy and practice. J Dev Eff 2012;4:409-429.

https://doi.org/10.1080/19439342.2012.710641

[35] Morray, B.H., Gordon, B.M., Crystal, M.A., Goldstein, B.H., Qureshi, A.M., Torres, A.J., Sathanandam, S.K. et al, 2020). Resource allocation and decision making for pediatric and congenital cardiac catheterization during the novel coronavirus SARS-CoV-2 (COVID-19) pandemic: A U.S. multi-Institutional perspective. The Journal of Invasive Cardiology, 32, $1-8$.

[36] Modi, A.C., Pai, A.L., Hommel, K.A., Hood, K.K., Cortina, S. Drotar, D. et al (2012). Pediatric Selfmanagement: A Model for Research, Practice, and Policy. Pediatrics, 129, e473-e485. DOI: 10.1542/peds.2011-1635.
[37] Nkonki L,L.L, Chola L,L, Tugendhaft A,A and Hofman, K.K. (2017). Modelling the cost of community interventions to reduce child mortality in South Africa using the Lives Saved Tool (LiST). BMJ Open 2017;7:e011425. doi:10.1136/bmjopen-2016-011425.

[38] Alkinaidri, A. and Alsulami, H. (2018). Improving Healthcare Referral System Using Lean Six Sigma. American Journal of Industrial and Business Management, 8, 193-206.

[39] Angelis, A., Kanavos, P. and Montibeller, G. (2017). Resource allocation and priority setting in health care: A multi-criteria decision analysis problem of value? Global Policy, 8 (2) doi: 10.1111/1758-5899.12387.

[40] Bowers, J., Cheyne, H., Mould, G., Miller, M., Page, M., Harris, F. and Bick, D. (2018). A multi-criteria resource allocation model for the redesign of services following birth. BMC Health Services Research, 18 (656), 1 - 12. 